\title{
Factores históricos, políticos y culturales en la transformación de Egipto en proveedor clave de cebolla deshidratada a Japón ${ }^{1}$
}

DOI: $10.32870 /$ mycp.v2i3.399

Carlos Javier Maya Ambía

Hossam Younes Abdel Aziz ${ }^{3}$

\section{Resumen}

En el presente trabajo se analiza la transformación de Egipto en uno de los principales proveedores de cebolla deshidratada a Japón, tratando de integrar en la explicación tanto factores de naturaleza económica como otros de carácter histórico, político y cultural para llegar a entender cómo es que un producto egipcio ha llegado a ocupar uno de los primeros lugares en el mercado de un país tan distante no sólo en términos geográficos.

Con historias muy diferentes, en ambos países la cebolla deshidratada ha llegado a cobrar gran importancia. En Egipto, como producto de exportación y en Japón como ingrediente esencial de una serie de alimentos procesados demandados de manera creciente por su población. Esta creciente demanda obedece de manera fundamental a cambios demográficos y en la cultura alimentaria de los japoneses. La incapacidad de Japón para abastecer su propia demanda, debido al tipo de desarrollo de su agricultura después de la II Guerra Mundial, explica las importaciones del producto estudiado, dentro de las cuales Egipto ha ido ganando terreno de manera notoria, en lucha con numerosos competidores, a los cuales ha sido capaz de ir desplazando. Esta

1. Artículo recibido el 22 de octubre de 2012 y dictaminado el 26 de noviembre de 2012.

2. Universidad Autónoma de Sinaloa. Correo electrónico: carlosmayaambia@hotmail.com.

3. Senior Researcher and Trade Facilitation Manager. Ministry of Industry \& Foreign Trade, Arab Republic of Egypt. Correo electrónico: h_Younes_1@yahoo.com. 
capacidad de los exportadores egipcios tiene su origen tanto en las tradiciones agrícolas del país, como en las políticas seguidas por los gobiernos egipcios.

Finalmente se presentan las experiencias, opiniones y valoraciones de directivos de las cinco mayores empresas egipcias exportadoras de cebolla deshidratada.

Palabras clave: Egipto, Japón, agroindustria, comercio internacional, cebolla deshidratada.

\section{Abstract}

In this paper we analyze the transformation of Egypt into one of the leading suppliers of dehydrated onions to Japan. The study introduces economical, historical, political and cultural factors, in order to understand how a product from Egypt, has become to occupy one of the first places in the Japan's market, a country so distant from Egypt not only in geographical terms.

Both countries have very different histories. However, in both of them dehydrated onions have become very important products. In Egypt it is an export item and in Japan it is a key ingredient of a great number of processed food items that are increasingly demanded in this country. This trend is due basically to demographic changes and to innovations in the food culture of the Japanese population. Shortcomings of the national production, due to the specific kind of development of Japan's agriculture after the Second World War, explain the imports of the studied product. In this scenario Egypt has been remarkably advancing, confronting and overcoming numerous competitors. This capacity of the Egyptian exporters has its origin in their agricultural traditions, as well as in the policies implemented by the Egyptian government.

Thereafter, we present the experiences, opinions and assessments of managers of the five most important Egyptian enterprises that are exporting dehydrated onions.

Key words: Egypt, Japan, agroindustry, international trade, dehydrated onions.

\section{Introducción}

Es nuestro propósito explicar la transformación de Egipto en uno de los principales proveedores de cebolla deshidratada a Japón, atendiendo no sólo 
a los factores económicos sino a los históricos, políticos y culturales que han contribuido a dicha transformación.

En primer lugar, se discute la relevancia del fenómeno estudiado. A continuación se busca identificar los contextos en los que ocurre este fenómeno, tomando en cuenta la ubicación tanto de Japón como de Egipto en el escenario de la actual globalización y la historia de dicha ubicación. En tercer lugar, se discute la relevancia de la industria procesadora de alimentos en ambos países y en particular la de la cebolla deshidratada, identificando los factores que han convertido a la industria egipcia de este producto en una de las principales del mundo; mientras que, por otra parte, se buscan los determinantes del incremento de la demanda de cebolla deshidratada en Japón así como de la insuficiencia en la producción doméstica. Este apartado se cierra con una revisión de las relaciones comerciales entre Japón y Egipto. El cuarto punto, corresponde al estudio de la competencia internacional en el mercado japonés del producto analizado. En el quinto apartado, se resumen los resultados de cinco estudios de caso. Se trata de información obtenida a partir de entrevistas y aplicación de cuestionarios a directivos de las mayores empresas egipcias productoras y exportadoras de cebolla deshidratada. El texto concluye con algunas consideraciones y reflexiones sobre la experiencia referida.

\section{Metodología}

El presente trabajo es producto de una labor de investigación realizada por los autores tanto en Japón como en Egipto. En Japón se realizaron una serie de entrevistas entre los meses de febrero y octubre de 2008, tanto con académicos como con empresarios y agricultores. En Egipto se aplicaron cuestionarios en septiembre de 2011 a cinco de las más importantes empresas exportadoras de productos deshidratados. Asimismo se consultaron las bases de datos sobre comercio internacional de la ONU (Comtrade) y la FAO, de la aduana japonesa y del gobierno egipcio, así como estudios y reportes sobre la industria procesadora de alimentos tanto japonesa como egipcia, realizados por estudiosos de diversos países. Complementariamente se realizaron consultas con otros especialistas radicados en México y Estados Unidos. 


\section{Objeto de estudio}

De acuerdo con datos de la FAO, en 2000 Japón era el octavo productor mundial de cebolla deshidratada, mientras que Egipto ocupaba el lugar número quince. Diez años después, según la misma fuente, Egipto aparece como el cuarto productor mundial y a Japón le corresponde el puesto número dieciocho (cuadro 1). Por otra parte, con base en registros de la aduana japonesa, en el primero de los años mencionados Egipto aportaba 3.95\% de las importaciones japonesas de cebolla deshidratada en términos de volumen, para llegar a alcanzar $18.11 \%$ en 2011. Debe señalarse que históricamente los principales proveedores del producto mencionado han sido EU y China, quienes han mantenido su posición, aunque con una reducción en el segmento del mercado correspondiente a EU y una fluctuante, pero a largo plazo constante participación de China, mientras que el tercer puesto lo ocupaba Rumania hasta 1994, pero al año siguiente lo pierde a favor de Egipto, cuando su segmento llega a un modesto $2.8 \%$, pero el de Rumania prácticamente desaparece. Si bien Egipto se ha mantenido desde entonces en el tercer puesto, su participación en el mercado se ha ampliado notablemente, a costa de los demás oferentes (cuadro 2).

Aunque a primera vista podría explicarse el fenómeno observado simplemente diciendo que la competitividad de los oferentes egipcios ha ido en aumento, mientras que probablemente tanto las de sus competidores como la de los propios fabricantes japoneses ha declinado o no ha aumentado al ritmo de la de los egipcios, esta explicación es insuficiente y nuestro propósito es averiguar qué otros factores convergen para arrojar el resultado arriba constatado. El hecho es que el mercado de cebolla deshidratada está mostrando varios fenómenos de interés, que mencionaremos a lo largo del texto, pero primero es necesario ubicar tanto a Japón como a Egipto en el contexto de la actual globalización y aclarar cómo es que han llegado a tales posiciones y a convertirse en socios comerciales.

\section{Japón y Egipto en el mundo globalizado}

En el contexto de la actual globalización (Altvater y Mahnkopf, 2002; Mittelman, 2000), Egipto representa un caso de país subordinado y Japón el de uno hegemónico, colocados cada uno históricamente en dos esferas geográ- 
Factores históricos, políticos y culturales en la transformación de Egipto en proveedor clave de cebolla deshidratada a Japón

Cuadro 1

Producción mundial de cebolla deshidratada (2000-2010)

\begin{tabular}{lrrlrr}
\hline Año 2010 & Toneladas & \multicolumn{1}{c}{$\%$} & Año 2000 & Toneladas & $\%$ \\
\hline China & 20507759 & 27,62 & 1 China & 14104696 & 28,29 \\
India & 13372100 & 18,01 & 2 India & 4721100 & 9,47 \\
EU & 3320870 & 4,47 & 3 EU & 3253190 & 6,52 \\
Egipto & 2208080 & 2,97 & 4 Turquía & 2200000 & 4,41 \\
Irán & 1922970 & 2,59 & 5 Pakistán & 1647990 & 3,31 \\
Turquía & 1900000 & 2,56 & 6 Irán & 1343590 & 2,69 \\
Pakistán & 1701100 & 2,29 & 7 Rusia & 1338660 & 2,68 \\
Brasil & 1556000 & 2,10 & 8 Japón & 1247000 & 2,50 \\
Rusia & 1536300 & 2,07 & 9 Brasil & 1141810 & 2,29 \\
R. Corea & 1411650 & 1,90 & 10 España & 959731 & 1,92 \\
Holanda & 1302000 & 1,75 & 11 México & 905452 & 1,82 \\
México & 1266170 & 1,71 & 12 R. Corea & 877514 & 1,76 \\
Myanmar & 1137900 & 1,53 & 13 Holanda & 821022 & 1,65 \\
Marruecos & 1131320 & 1,52 & 14 Indonesia & 772880 & 1,55 \\
Argelia & 1111200 & 1,50 & 15 Egipto & 762993 & 1,53 \\
España & 1106900 & 1,49 & 16 Colombia & 745604 & 1,50 \\
Indonesia & 1048230 & 1,41 & 17 Polonia & 720345 & 1,44 \\
Japón & 1047000 & 1,41 & 18 Nigeria & 600000 & 1,20 \\
Total & 74250809 & 100 & Total & 49863348 & 100 \\
\hline
\end{tabular}

Fuente: Faostat

ficamente distintas de la globalización, pero que de tiempo atrás han estado buscando diversificar sus nexos con otras economías.

Durante el siglo XIX, Egipto sufre el peso colonial de Francia, Inglaterra y el Imperio Otomano. Debilitado este último, Egipto obtiene su independencia en 1936, pero se mantiene la presencia británica (Lufti y Marsot, 2007; Thompson, 2008). En 1952 una sublevación acaba con la monarquía egipcia y se inicia la república, encabezada por Nasser desde 1954, quien al nacionalizar el Canal de Suez se coloca como enemigo de Francia y Gran Bretaña y busca la protección de la URSs, con la que desarrolla una intensa actividad comercial. Durante el periodo de Nasser se habla de un socialismo árabe que conlleva una fuerte intervención estatal en la economía. En 1970 muere Nasser y ocupa la presidencia Sadat, quien promueve la liberalización 


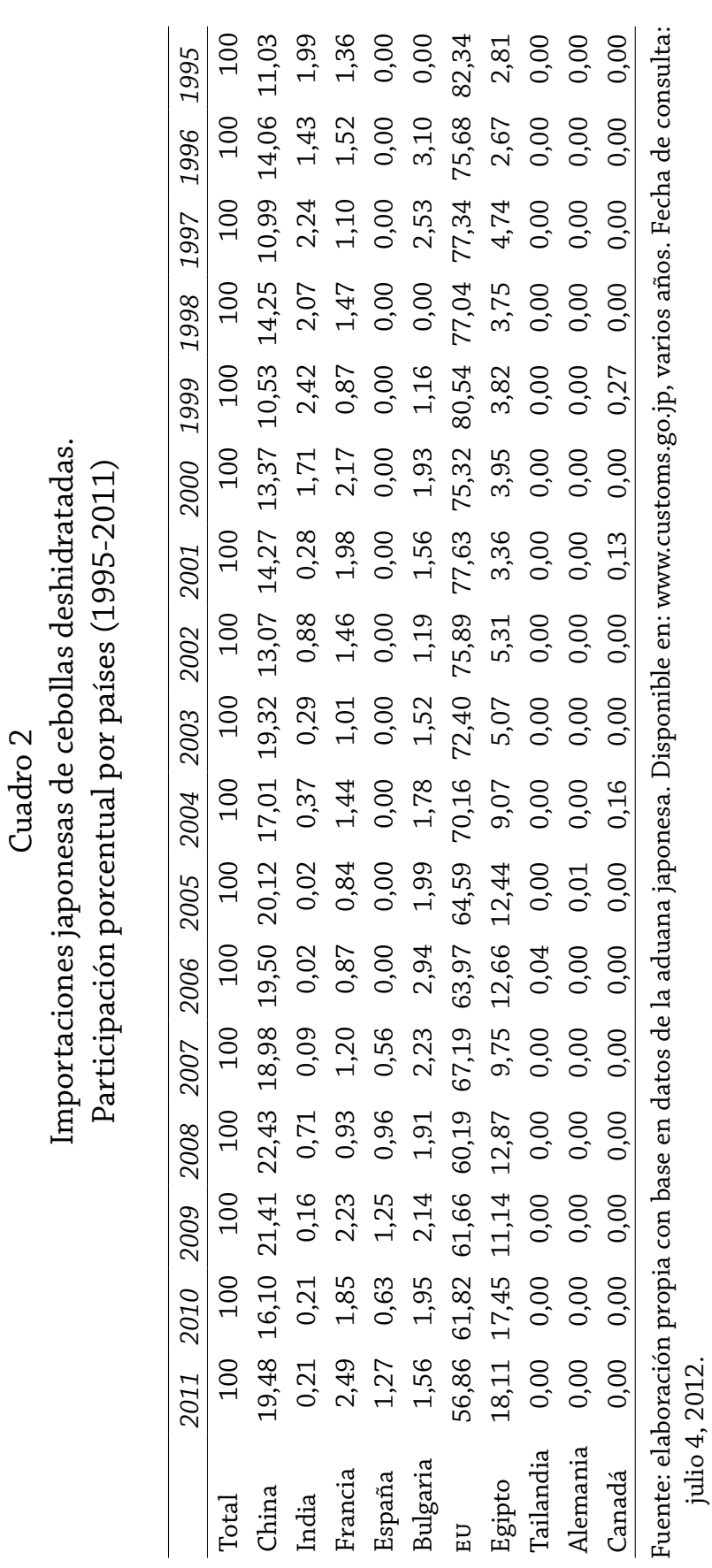


económica y busca un acercamiento con EU y con los países europeos, hasta 1980, cuando es asesinado. El cargo queda en manos de Mubarak, quien se mantiene en el poder durante más de tres décadas, durante las cuales estrecha las relaciones con EU y Europa occidental, pero al mismo tiempo mantiene intensos flujos comerciales con los demás países árabes. En este periodo se agudiza la dependencia económica de Egipto de sus ventas petroleras, que siguen siendo la principal fuente de divisas por concepto de exportaciones. En cambio el sector agrícola es uno de los más débiles, pues sólo entre $4 \%$ y $5 \%$ de la superficie del país es tierra cultivable; esto obliga a la importación de alimentos, las cuales representan entre $10 \%$ y $15 \%$ de sus importaciones totales. Sin embargo, el país produce suficientes frutas y hortalizas para satisfacer la demanda doméstica e incluso exportar algunos productos como papas y cítricos. Dentro del sector industrial egipcio han sobresalido tres industrias: hidrocarburos, textil y alimentos procesados. Estas industrias se han orientado a los mercados externos y dentro de la última destacan las exportaciones de cebollas deshidratadas, seguidas por las de verduras congeladas, jugos de frutas, hierbas y especias, así como quesos.

Los principales destinos de las exportaciones egipcias de alimentos procesados son los países ubicados alrededor del Golfo Pérsico, así como en el sudeste de la cuenca del Mediterráneo, a los que se destina aproximadamente $45 \%$ de las ventas externas de dichos productos. Siguen en importancia las exportaciones a los países de la Unión Europea, quedando en tercer lugar los países norafricanos y Estados Unidos.

Por lo que concierne a Japón, aunque llega tardíamente al reparto colonial del mundo entre las grandes potencias occidentales, jamás fue colonia y conoce una acelerada modernización económica y política durante la segunda mitad del siglo XIX (véase Hane, 2003). A principios de la siguiente centuria muestra tendencias imperialistas que ubican sus intereses económicos en los países de su entorno geográfico, lo cual desemboca inevitablemente en confrontaciones con las potencias ya anteriormente asentadas en dichas regiones. Dentro de esta dinámica es que se llega a la II Guerra Mundial, a la derrota de Japón y sus aliados y a la eventual ocupación del país por parte del ejército de Eu, bajo el mando del general McArthur, quien con sus profundas reformas económicas transforma al país y promueve una modernización que en otras condiciones habría necesitado décadas para realizarse (véase Tsuru, 1999). Sobre esta base es que Japón alcanza un extraordinario crecimiento económico que continúa hasta la década de 1990, cuando se hunde en una crisis cuyas repercusiones 
todavía se sienten en la actualidad. En este contexto se va convirtiendo Japón en un nuevo centro hegemónico de la economía globalizada, con una fuerte presencia en el comercio internacional, sobre todo en industrias que llegan a ser la vanguardia de los avances tecnológicos del siglo $\mathrm{xx}$, como son la automotriz y la electrónica. Sin embargo, en general el sector industrial japonés se beneficia de los logros tecnológicos para aumentar su productividad.

Por otro lado, en buena medida a raíz de la política económica del general McArthur - quien se fijó como uno de sus objetivos acabar con las grandes propiedades agrícolas en Japón, pues en ellas veía al más fuerte sostén del militarismo japonés-, la agricultura nipona se va rezagando con respecto al resto de la economía. De tal suerte que las unidades agrícolas son en su mayoría de pequeña escala, a lo que se suman dos hechos que han sido de enorme importancia. El envejecimiento de la población dedicada a la agricultura y la ausencia de un relevo generacional, dado que los hijos de los agricultores no han estado dispuestos a continuar la carrera de sus padres y abuelos, sino que han preferido calificarse como obreros y empleados para los sectores industrial y de servicios. En este contexto, la industria japonesa procesadora de alimentos ve su suerte estrechamente ligada a la de la agricultura, pero al volverse insuficiente la oferta agrícola doméstica, tiene que echar mano de las importaciones provenientes de países con sectores agrícolas más vigorosos.

Como potencia comercial moderna, Japón tuvo sus primeros vínculos con países asiáticos, pero a raíz de la segunda posguerra estos vínculos se enfocan con fuerza hacia EU y después hacia Europa occidental, quienes actualmente son los principales socios comerciales de dicho país. Sin embargo, si agrupamos a todos sus socios comerciales asiáticos, a la cabeza de los cuales se encuentra China, encontramos que los principales intereses comerciales nipones se ubican en el este asiático, lo cual confirma el hecho de que Japón, junto con China se ha convertido en uno de los tres grandes centros hegemónicos de la economía global.

Con lo anteriormente expuesto, podemos darnos cuenta de que Egipto y Japón se localizan en distintos espacios económicos y que ninguno de estos países se encuentra en la lista de los principales socios comerciales del otro. Sin embargo, a nivel de productos específicos, hay indicios de que ambos países han diferenciado sus relaciones comerciales, como lo muestra el caso de las importaciones japonesas de cebollas deshidratadas, lo cual nos lleva a ocuparnos a continuación de la industria procesadora de alimentos en Japón y en Egipto. 


\section{La industria procesadora de alimentos en Japón y en Egipto}

La industria procesadora de alimentos en Japón es la tercera más grande del país después de la electrónica y de la automotriz $\mathrm{y}$ seis veces mayor que la de EU, si se toma como referencia la población de cada país, y ocupa aproximadamente $11 \%$ de la fuerza de trabajo empleada en el sector manufacturero. Aunque tradicionalmente ha estado compuesta en su mayoría por pequeñas y medianas empresas, ${ }^{4}$ que han soportado excesivas regulaciones gubernamentales y acostumbracionismo, por lo que su productividad total ha estado muy por debajo de la de sus homólogos estadounidenses, ${ }^{5}$ al mismo tiempo se trata de un sector altamente concentrado, pues está dominado por 15 grandes empresas que manejan alrededor de $50 \%$ de las ventas, ${ }^{6}$ mientras el resto corresponde a una gran cantidad de productores pequeños de baja eficiencia y competitividad. ${ }^{7}$

4. Más de $80 \%$ de las empresas procesadoras de alimentos ocupan menos de 300 empleados.

5. Véase "Japan's food industry" en: The Economist, agosto 17, 2000.

6. Entre las mayores empresas que emplean cebolla deshidratada en la elaboración de algunos de sus productos pueden mencionarse las siguientes: Kirin, Meiji, Maruha, Nippon Meat Packers, Ajinomoto, Yamazaki, Morinaga, Nichirei, Nisshin Seifun Group, Itoham Foods, Nissin Foods, Nippon Suisan Kaisha, Nissin Olio Group, Kikkoman Corporation, Kagome, Mizkan, por señalar a las más importantes (véase Aoki y Miyamoto, 2011).

7. Debe precisarse que aún las grandes empresas del sector son pequeñas en comparación con las grandes empresas japonesas en otras industrias. Por ejemplo, las grandes empresas electrónicas emplean entre 60,000 y 70,000 trabajadores, mientras que sus homólogas del sector alimentario cuentan con 7,000 trabajadores (véase Itoh e Ito, 2003). Asimismo, las grandes empresas japonesas procesadoras de alimentos son pequeñas en comparación con las estadounidenses, lo cual se debe, entre otras razones, a que en Japón han estado legalmente prohibidas las adquisiciones y fusiones de empresas consideradas grandes por las autoridades gubernamentales. 
Por lo que toca a la demanda de alimentos procesados, ésta ha estado creciendo con especial fuerza durante la última década y actualmente los alimentos procesados representan aproximadamente dos tercios del consumo de alimentos en Japón y hasta $90 \%$ si se incluyen las comidas fuera de casa y otros servicios alimentarios. La creciente demanda de alimentos procesados se debe a varios factores, como son una mayor preocupación de la población por una alimentación saludable y segura, así como una diversificación de la dieta, ${ }^{8}$ un aumento de la población de más de 65 años y una mayor participación de las mujeres en la fuerza de trabajo asalariada. También debe señalarse que los jóvenes japoneses tienden a casarse más tarde que antes, ${ }^{9}$ razón por la cual hay cada vez más japoneses que cocinan o se preparan sus alimentos para ellos mismos, ${ }^{10}$ muchos de los cuales adquieren ya empacados en las 42,000 tiendas de conveniencia ubicadas en todo el país. ${ }^{11}$

Es preciso apuntar que los productores de alimentos han hecho diferenciaciones demográficas entre los consumidores. La más evidente y probablemente la más antigua sea la de los niños, que consumen cierto tipo de alimentos preparados. Después ha venido la de las personas mayores de 65 años, quienes, como ya se apuntó constituyen en Japón un grupo etario de creciente presencia, ${ }^{12}$ con sus propias preferencias alimentarias. Posteriormente se identificó como un grupo especial de consumidores a las mujeres de 40 años, quienes preferentemente preparan sus alimentos en sus hogares. Más recientemente se ha constituido un nuevo grupo conformado por hombres y mujeres entre los 20 y 30 años. Estos jóvenes ya no viven con sus padres, pero tampoco se han casado y buscan alimentos ya preparados o de fácil preparación, presentados en porciones para una sola persona..$^{13}$ Entre las preferencias de estos consumidores destacan las pastas de sencillo cocimiento,

8. Cuando mencionamos diversificación de la dieta nos referimos al incremento en el consumo de los japoneses de alimentos provenientes de EU y Europa, como pizzas, hamburguesas, pollo frito que han sido masivamente promovidos por empresas como McDonald's, Domino's Pizza, KFC, Starbucks, por mencionar a las más conocidas.

9. Actualmente la edad promedio de matrimonio de las mujeres es de 30 años y la tasa de matrimonio es de 5.7 por cada 1,000 personas.

10. Hablamos tanto de hombres como de mujeres.

11. Véase Bestor (2006) y Whitelaw (2006).

12. En el año 2011 representaron ya el $23.3 \%$ de la población y se estima que para fines de la presente década habrá tres japoneses adultos mayores por cada niño menor de 15 años.

13. De hecho el porcentaje de hogares unipersonales en el total de hogares japoneses ya representaba $23 \%$ en 2000 y esta cifra ha venido aumentando desde entonces, para alcanzar $31.5 \%$ en 2011 y se estima que llegará a 34.5\% en 2020 (datos de Euromonitor International. Disponible en: http:// 
acompañadas de sus correspondientes salsas. Por otra parte, los productores de alimentos preparados han observado que este grupo de consumidores responde sensiblemente a la forma y colorido del empaque del producto; saben claramente qué quieren consumir sin que el precio sea decisivo y, algo muy importante, se muestran leales a las marcas de los productos elegidos.

Estos factores han elevado la demanda de alimentos listos para consumirse, así como ofrecidos en presentaciones más convenientes para los consumidores. Esta creciente demanda ha hecho posible que a pesar de la recesión, la industria procesadora de alimentos haya tenido un desempeño superior al de la economía japonesa en general. Por parte de los procesadores de alimentos debe señalarse que el entorno deflacionario imperante en Japón en los últimos años, los ha llevado a buscar insumos a bajo costo en los mercados internacionales y a explorar opciones de procesamiento en el extranjero para seguir siendo competitivos. ${ }^{14}$ Pero también las exigencias de los consumidores han llevado a los fabricantes a mostrarse muy estrictos con sus proveedores en cuanto a seguridad e inocuidad alimentaria, respeto de normas y estándares sanitarios y fitosanitarios, calidad y rastreabilidad del producto y a exigirles a sus proveedores la aplicación de sistemas como HACC. ${ }^{15}$

Dentro de la industria mencionada, la procesadora de frutas y verduras es la que ha mostrado un mayor crecimiento. Incluso algunos alimentos instantáneos, salsas de soya y condimentos manufacturados en Japón son exportados. De ahí la necesidad de incrementar las importaciones de los insumos para la fabricación de estos productos, como es el caso de la cebolla deshidratada.

Por lo que concierne a la industria procesadora de alimentos en Egipto, debemos observar lo siguiente.

En realidad el procesamiento de alimentos tiene una historia milenaria en Egipto, pues ya hace más de 4,500 años se elaboraba cerveza, cuyo origen se atribuye al dios Osiris. Sin embargo, para los propósitos de nuestra investigación es suficiente remontarnos a mediados del siglo xx, cuando con capital

blog.euromonitor.com/2012/03/single-person-households-on-the-rise-in-japan.html. Fecha de consulta: agosto 26, 2012).

14. Se trata de la llamada producción off-shore. Por ejemplo, Ajinomoto, que es la cuarta empresa más grande de alimentos congelados en Japón, tiene siete plantas manufactureras en el extranjero. Nippon Ham, la mayor empresa procesadora de carne en Japón tiene joint-ventures en Australia, México y EU. Asimismo numerosas empresas japonesas continúan invirtiendo en China para producir alimentos destinados al mercado japonés (información de Aoki y Miyamoto, 2011).

15. Sistema de Análisis de Peligros y Puntos Críticos de Control, por sus siglas en inglés. 
extranjero se instalan las primeras plantas deshidratadoras de verduras en Puerto Said, ciudad ubicada al nordeste del país a orillas del mar Mediterráneo. Estas inversiones trazaron la ruta para el crecimiento de futuros mercados, como ha sido la fabricación industrial de ingredientes para sopas y salsas y en general para los negocios de comida rápida. En la década de 1960 se inició el procesamiento de cebollas deshidratadas al sur de El Cairo y posteriormente se erigieron tres nuevas fábricas en Alejandría y en el área del Delta del Nilo. Actualmente existen ocho plantas deshidratadoras localizadas en el triángulo geográfico de El Menia-Puerto Said-Alejandría, cuyas distancias promedio hasta las granjas proveedoras de sus insumos es de aproximadamente 50 kilómetros, aunque en particular las cebollas pueden provenir de granjas ubicadas a mayores distancias. Hoy en día Egipto es uno de los mayores productores mundiales de verduras deshidratadas, con un volumen total de aproximadamente dos millones de toneladas, combinando la más moderna tecnología con los métodos tradicionales de secado al sol. Las cebollas deshidratadas son el principal producto, pues ocupan $28 \%$ del área total de cultivo de verduras para deshidratación y su producción representa $80 \%$ del total de verduras deshidratadas. Por otra parte, Egipto tiene una larga tradición en el cultivo de la cebolla, realizado principalmente en el sur del país, con volúmenes de aproximadamente 750 mil toneladas anuales, de las cuales alrededor de 140 mil toneladas se exportan. Debido a la expansión de la industria deshidratadora de cebollas, ha aumentado el cultivo de variedades adecuadas para su deshidratación, como la llamada "globo blanco", pues conservan su color, sabor y otras propiedades aún después de ser procesadas industrialmente. Como en Egipto las cebollas pueden cosecharse dos veces al año, las plantas deshidratadoras trabajan aproximadamente 10 meses al año. Junto con las cebollas, el principal producto deshidratado son las habas y varios tipos de leguminosas. En cuanto a exportaciones de verduras deshidratadas también las cebollas han ocupado el primer lugar, seguidas de alubias o habichuelas y en tercer lugar habas. En menores cantidades igualmente se exportan lentejas, garbanzos y otras leguminosas. En la segunda mitad de la primera década del presente siglo, el valor de las exportaciones de los productos mencionados casi se triplicó, llegando a 130 millones de USD en 2010. De la misma manera, Egipto ha empezado a exportar productos orgánicos a países desarrollados.

Los principales destinos de las exportaciones egipcias de verduras deshidratadas son Alemania (26.3\%), Japón (10.1\%), Holanda (9.5\%), Bélgica (8.7\%), Gran Bretaña (5.7\%) y EU (4.9\%). Dentro de estos productos desta- 
can las cebollas deshidratadas, de las cuales en 2010 se exportaron 48,871 toneladas, destinándose 3,957 a Alemania; 3,655 a Japón; 1,431 a Holanda; 1,358 a Bélgica y 955 a Gran Bretaña. ${ }^{16}$ También se deshidratan especias como albahaca, comino, tomillo, menta, manzanilla y otras. Algunas de las empresas líderes tienen fuertes lazos con compañías extranjeras, como McCormick, Kato y firmas alemanas. Ciertamente en las últimas dos décadas se ha incrementado la producción de diversas verduras deshidratadas en Egipto, pero el principal producto sigue siendo la cebolla deshidratada, ${ }^{17}$ cuya producción se ha venido incrementando constantemente hasta superar las 14 mil toneladas, ${ }^{18}$ de las cuales aproximadamente 10,000 toneladas se exportan a la Unión Europea, en particular a Alemania, Holanda y Gran Bretaña, donde se concentra $90 \%$ de estas ventas. Aunque los precios pagados por los compradores europeos resultan atractivos para los exportadores egipcios, ${ }^{19}$ los volúmenes procesados dependen también de los precios de las cebollas frescas en los mercados internacionales. Si estos precios son mayores, entonces es preferible exportar el producto en fresco.

Es importante destacar que actualmente cuatro de las mayores empresas deshidratadoras han realizado grandes inversiones para lograr una automatización completa del proceso de deshidratación, de tal suerte que se puedan procesar 14 toneladas por día. Estas inversiones proceden básicamente de programas de créditos blandos de USAID (United States Agency for International Development) y la tecnología aplicada proviene también de EU. Estas inversiones incluyen detectores de metales y clasificadores por colores que permiten mejorar la calidad del producto para satisfacer las exigencias de los clientes industriales, tanto europeos como de otros países. Una reducida parte de la producción se destina al pequeño mercado nacional, compuesto por fabricantes de sopas, salsas y pasta de tomate.

16. Alubias y garbanzos son también importantes productos de exportación, pero los principales mercados para Egipto son Irak, Siria, Libia, Rumania y Túnez. En términos de valor las exportaciones de estos productos superan a las de cebollas deshidratadas (42.1 millones de dólares contra 31.7 millones de dólares en el año 2010).

17. El segundo producto seco más importante son las habas, seguidas de los frijoles.

18. No hay datos oficiales al respecto, sólo estimaciones a partir de informes de las propias empresas. Véase: Tezera et al. (2005).

19. Por ejemplo 2,000 USD por kilogramo de polvo fino de cebolla deshidratada. 
Asimismo, debe enfatizarse que el gobierno egipcio brinda apoyo a la industria procesadora de alimentos a través de varios mecanismos, entre los que destacan los tres que se detallarán a continuación.

El primero consiste en soporte institucional por medio del Consejo de Exportaciones y de la Cámara de la Industria Procesadora de Alimentos. Estos dos organismos respaldan a sus miembros para participar en ferias nacionales e internacionales en colaboración con la Asociación de Exportadores Egipcios (Expolink); promueven viajes de misiones comerciales al extranjero; proporcionan a sus miembros reportes e información estadística concernientes a sus actividades; apoyan a sus miembros para mejorar su competitividad y obtener certificados como ISO y otros; brindan cursos de entrenamiento y capacitación; y fomentan la creación de sitios Web para las empresas miembro, así como facilitan su participación en sitios Web internacionales.

El segundo canal de apoyo lo constituye el Centro de Modernización Industrial (IMC, por sus siglas en inglés), que brinda asistencia técnica y programas de capacitación para aumentar la competitividad del sector de procesamiento de alimentos.

La tercera vía consiste en recursos financieros procedentes de un fondo para el desarrollo de las exportaciones. Se trata de una iniciativa nacional fundada por el gobierno egipcio para promover el desarrollo de la competitividad internacional del sector manufacturero privado, para beneficiarse de las nuevas oportunidades brindadas por los mercados globales. Este apoyo financiero constituye aproximadamente $10 \%$ del valor agregado de las exportaciones. ${ }^{20}$

En estas condiciones las cebollas deshidratadas encabezan las exportaciones egipcias de alimentos. No sólo se han consolidado en sus mercados tradicionales, sino también han venido conquistando nuevos mercados, como Rusia y Japón, desplazando probablemente a exportadores europeos. En opinión de las empresas extranjeras clientes de los exportadores egipcios, la cebolla deshidratada egipcia posee muy alta calidad y precios competitivos. Gracias a ello, a lo largo de la primera década del presente siglo, Egipto se ha posicionado como el primer proveedor externo de la Unión Europea, con un segmento del mercado cercano a $33 \%$, mientras que a principios del lapso señalado era de $20 \%$. En estos años Egipto ha desplazado en el mercado europeo a EU como primer proveedor y ha ampliado su distancia frente a China. India también ha ganado terreno en Europa gracias a sus precios competiti-

20. Información directa del Ministerio de Comercio e Industria de Egipto. 
vos, pero sus productos son de menor calidad y grado de estandarización que los egipcios. Sin embargo, ya en el periodo mencionado era evidente que los mercados maduros de Europa occidental mostraban un lento crecimiento, por lo que era recomendable avanzar en otros mercados en expansión, como era el caso de los países de Europa del este y Japón. ${ }^{21}$

El principal insumo de la cebolla deshidratada proviene de la agricultura. El producto se ubica en la industria procesadora de alimentos y su destino es esta misma industria, ya que la cebolla deshidratada es un ingrediente fundamental en una serie de alimentos procesados, como sopas, salsas, purés, pizzas y hamburguesas, por mencionar los más relevantes.

Al principio de nuestro trabajo, señalamos que hacia 2000 Japón era el octavo productor mundial de cebolla deshidratada, pero que a lo largo de la década la situación cambió radicalmente. Nuestro propósito es indagar las causas de este cambio y sus consecuencias.

En todas las entrevistas realizadas en Japón, se nos informó que aunque la agricultura japonesa mostraba una tendencia históricamente declinante, con la agroindustria estaba ocurriendo justamente lo contrario; de ahí que para países exportadores de frutas y verduras, como México, resultara interesante convertirse en proveedores no sólo de productos frescos sino con algún grado de procesamiento, para funcionar como insumos de la mencionada industria, pues alrededor de $80 \%$ de las verduras deshidratadas empleadas en la industria japonesa son importadas. Por otra parte, lo constatado anteriormente con relación al procesamiento de cebolla en Japón nos lleva a pensar que dentro de la industria japonesa de alimentos procesados se han presentado desarrollos dispares; algunas ramas han crecido con fuerza y otras, como es el caso de la industria de la cebolla deshidratada, se han colapsado.

En las siguientes líneas trataremos de explicar las razones de este colapso.

Sin duda Japón tuvo una producción doméstica importante de cebolla deshidratada hasta el año 2000, pero incluso antes de esa fecha, dicha producción ya era insuficiente para abastecer la demanda de las empresas procesadoras de alimentos; por lo que a lo largo del siglo xx se realizaron importaciones de EU, cuya industria de cebolla deshidratada empieza a cobrar grandes dimensiones después de la II Guerra Mundial, debido a las necesidades de

21. Información procedente de Pinard, Scotti, Ahmed (2006). Aquí cabe observar que Egipto no destaca en los mercados internacionales (Europa y EU) con otros productos deshidratados. Tanto en Europa como en EU, el líder es China. 
reconstrucción industrial, tanto en Europa como en Japón. De ahí que, las exportaciones estadounidenses se canalizaran masivamente hacia esos países, cuyas industrias deshidratadoras de verduras habían quedado destrozadas por el conflicto bélico. ${ }^{22}$ Desde entonces, EU se ha mantenido como el principal proveedor externo de cebolla deshidratada a Japón, aunque su participación ha disminuido de $82.3 \%$ en 1995 a $56.8 \%$ en 2011, mientras que se ha incrementado la presencia de otros proveedores como China, India en algunos años, Francia y notablemente Egipto. Las compras a estos países obedecen a que la producción doméstica japonesa es insuficiente para satisfacer la creciente demanda, debido fundamentalmente a dos factores. El primero de ellos son los altos costos laborales en la industria, imperantes en Japón. El otro factor decisivo han sido los costos materiales. ${ }^{23}$ Debe recordarse que las tres principales zonas de cultivo de cebolla en Japón se ubican, en orden de importancia, en Hokkaido, Hyogen y Saga, correspondiendo a Hokkaido más de la mitad de la producción nacional. ${ }^{24}$ Sin embargo, la superficie cultivada se ha ido reduciendo desde la segunda mitad de la década de 1990 debido a escasez de mano de obra y a los bajos precios en el mercado nacional, que merman la rentabilidad de los cultivos. La insuficiente producción nacional de cebolla ha tenido que compensarse con importaciones de cebolla fresca, que fueron aumentando de 150,000 toneladas en el año 2002 hasta más de 350,000 toneladas en el año 2005. Desde entonces, los volúmenes importados han ido descendiendo hasta aproximadamente 200,000 toneladas en el año 2009. Los principales proveedores de cebolla fresca en el año 2002 fueron China y EU, aportando $46 \%$ y $42 \%$ de las importaciones respectivamente. Sin embargo, para 2009 China ya aportaba $84 \%$ y Eu sólo $11 \%$. También se han importado cebollas congeladas, mayoritariamente de China. Por lo que toca a las cebollas deshidratadas, las importaciones han aumentado de 5,023 toneladas en el año 2002 a 5,613 toneladas en el año 2009. La aportación de EU pasó de $76 \%$ a $62 \%$; la de China subió de $13 \%$ a 21\% y la de Egipto se elevó de $5 \%$ a $11 \%$ (información proveniente de ALIC, 2011).

Por otra parte, el crecimiento de la demanda japonesa de cebolla deshidratada obedece fundamentalmente a la expansión de la industria procesadora

22. Agradezco esta información al señor Juan Villarreal.

23. Información proporcionada por el señor Kazumi Suhara.

24. El cultivo de cebolla se inició de manera experimental en Sapporo en el año de 1871 y en 1878 ya se producía comercialmente. En Hokkaido la cebolla se siembra en febrero-marzo, se trasplanta en mayo y se cosecha en septiembre (véase Araki, 2009). 
de alimentos entre cuyos insumos se encuentra la cebolla deshidratada. Nos referimos básicamente a la producción de sopas, pastas tipo "noodles", salsas y en general comida instantánea, cuya elaboración requiere de manera constante a lo largo de todo el año el suministro de sus insumos, y no solamente en las épocas de cosecha en Japón.

Sobre las relaciones comerciales entre Japón y Egipto, debemos recordar que estos dos países han participado en mercados mundiales diferentes aunque coinciden en algunos, como el estadounidense, pero con mercancías muy distintas-. Japón es un exportador de productos industriales de alta tecnología, mientras que Egipto lo es principalmente de petróleo y con amplio margen de diferencia, de textiles y algunos productos alimenticios. Ambos países han mantenido relaciones diplomáticas cordiales y entre todos los países árabes, Egipto es la nación con la que Japón mantiene las mejores relaciones diplomáticas. Sin embargo no existe correspondencia con las relaciones económicas, aunque desde 2008 el comercio bilateral se ha incrementado notablemente en comparación con los niveles de las décadas anteriores. En este rubro Japón ha mantenido un superávit comercial frente a Egipto, cuyas principales exportaciones a la nación asiática han sido mayoritariamente petróleo y sus derivados (80.7\%), seguidas de bienes manufacturados (6.8\%) y alimentos (6.1\%), mientras que las exportaciones niponas a Egipto comprenden fundamentalmente maquinaria y equipo de transporte $(68.5 \%)$ y bienes manufacturados (19.3\%). ${ }^{25}$

En el sector de verduras deshidratadas existe complementariedad entre ambos países, pues Japón representa un gran mercado para estos productos, ${ }^{26}$ mientras Egipto es un productor y exportador líder en varios de estos alimentos, en particular cebolla deshidratada, cuyas exportaciones a Japón sumaron 4.8 millones de USD en el año 2010.

25. Datos de Un, Comtrade Database 2011.

26. En 2010 Japón importó verduras deshidratadas por aproximadamente 490 millones de USD, frente a 281 millones de USD diez años antes y arrojando en volumen un crecimiento de $74.4 \%$ en el mismo lapso (datos de UN, Comtrade). 


\section{La competencia internacional en el mercado japonés de cebollas deshidratadas}

Japón es uno de los principales compradores mundiales de alimentos; en particular, su industria procesadora también realiza importaciones de insumos de grandes dimensiones.

Las estadísticas de las oficinas aduanales japonesas nos permiten rastrear hasta 1988 las importaciones del producto que estamos estudiando. Desde entonces, hasta la fecha encontramos que Japón ha importado cebollas deshidratadas de 21 países, entre los que se encuentran tanto naciones altamente industrializadas, como eU, Francia, Alemania y Canadá — por mencionar algunos ejemplos-, como países de menor desarrollo, como Thailandia, Indonesia, Vietnam, Egipto y México.

Por otra parte, se constata que la mayoría de los 21 países que en algún momento han exportado cebollas deshidratadas a Japón, lo han hecho de manera discontinua. Algunos de ellos aparecen como proveedores unos cuantos años, como Hungría, México y Holanda, y luego desaparecen del mercado. También hay países, como Rumania, que estuvieron presentes una o más décadas y después se ausentan. Asimismo, se identifican recientes incursiones en el mercado, como es el caso de España, cuyos productos se registran desde 2007. En el extremo opuesto encontramos proveedores constantes, como China, India, Francia, Bulgaria, EU y Egipto. Actualmente los principales proveedores externos del producto estudiado son, en orden de importancia, EU, China y Egipto, con segmentos del mercado de $56.8 \%, 19.4 \%$ y $18.1 \%$ respectivamente para el año 2011. Es decir, que estos tres países aportan más de $94 \%$ de las importaciones realizadas por Japón. Sin embargo, es importante destacar que la participación de EU, quien sigue siendo el principal proveedor, ha venido reduciéndose continuamente, después de haber tenido segmentos del mercado por encima de $80 \%$ en algunos años (1995 y 1999). La participación de China en promedio para los 24 años observados ha sido de $16.3 \%$, con fluctuaciones en algunos años; por su parte, la de Egipto ha registrado los mayores incrementos, pues comenzó con $3.2 \%$ en 1988 , se redujo hasta $1.4 \%$ en 1994, para crecer de manera sostenida hasta alcanzar el actual $18.1 \%$ (véase cuadro 2). De tal manera que si las tendencias se mantienen, posiblemente pronto Egipto se convierta en el segundo proveedor de cebolla deshidratada después de EU, aunque superar a China no será tarea fácil. 
Asimismo, se trata de un mercado con una alta concentración, pero donde la competencia ha sido muy intensa y existen competidores con amplias posibilidades de expansión debido a su gran producción doméstica, como es el caso de India.

Por otra parte, la entrada o salida del mercado japonés por parte de los grandes productores mundiales de cebolla deshidratada, depende también del comportamiento de sus mercados domésticos y en la medida que en sus propios países se desarrolle una fuerte industria procesadora de alimentos, es factible que sus productos se conviertan en insumos de sus connacionales. Además, como ya se ha observado, la producción de cebolla deshidratada se ve afectada por los precios que alcancen en los mercados las cebollas frescas, de tal suerte que el incremento en estos precios puede desalentar la deshidratación de las liliáceas.

A continuación veremos cómo se presentan los fenómenos y tendencias arriba señalados a nivel de empresas específicas. Para ello presentamos los siguientes estudios de caso.

\section{Estudios de caso}

Se aplicaron cuestionarios y se realizaron entrevistas con directivos de cinco de las mayores empresas egipcias de verduras deshidratadas, cuyos nombres mantendremos en el anonimato.

Además de exportar a Japón, estas empresas exportan a Gran Bretaña, Italia, Alemania y otras naciones europeas, del Golfo Pérsico y países árabes y EU. Las ventas a Japón son relativamente recientes, pues una empresa lleva sólo dos años realizándolas y otra ya tiene siete años con estas operaciones, mientras que las demás declararon cinco años.

En la mayoría de los casos el acceso al mercado japonés se logró con asistencia de JETRO (Japan External Trade Organization), organismo del gobierno japonés para la promoción de las relaciones económicas con otros países. En este caso los entrevistados recibieron apoyo de JETRO para asistir a ferias y eventos agrícolas y hortícolas celebrados en Japón. Un entrevistado mencionó también el apoyo del gobierno egipcio a través del Egyptian International Trade Point, organismo encargado de la promoción del comercio exterior y que brinda orientación a las empresas interesadas en exportar sus productos.

Los entrevistados declararon que decidieron incursionar en el mercado japonés debido a lo atractivo de los precios, que compensan las exigencias 
de alta calidad de los productos. Uno de los entrevistados agregó que en las transacciones con empresas japonesas existe mayor transparencia que en otros casos y dos de ellos consideran que el mercado japonés es prometedor.

Ninguna de las empresas estudiadas exporta a Japón solamente cebollas deshidratadas, sino también otros productos, como verduras congeladas, diversas variedades de frijoles, dátiles, palmito, lentejas e incluso en un caso naranjas y algodón. Sin embargo, los principales productos exportados a Japón son, junto con las cebollas deshidratadas, dátiles y frijoles. Todos estos productos se transportan en avión a Japón y llegan entre 2 y 7 días a las empresas importadoras japonesas, aunque la ruta es directa entre ambos países.

Entre las dificultades que enfrentan, un entrevistado señaló que los costos de transporte son muy superiores a los de sus competidores ubicados en países más cercanos a Japón. Otro apuntó que sólo en una ocasión su envío fue devuelto porque el empaque no satisfacía las exigencias de los japoneses. Uno más comentó que una vez su producto fue rechazado por no cumplir con las exigencias de limpieza de los compradores.

Todos los entrevistados coincidieron en que sus ventas a Japón han aumentado. Cuatro apuntaron que los incrementos han sido tanto en volumen como en valor y uno de ellos señaló que el incremento había sido sólo en valor, pero no en cantidades. Sin embargo, estas ventas representan bajos porcentajes dentro de las exportaciones totales de las empresas analizadas. Para tres de ellas representan 5\%; para una 3\% y para otra $8 \%$. Por otra parte, como es usual en el mercado japonés, la distribución de estos productos dentro del país queda en manos de alguna empresa comercializadora nipona (trading companies), con la que se estableció contacto en alguna feria internacional celebrada en Japón, o bien a través de las oficinas de JETRO en El Cairo, o por medio del Egyptian International Trade Point.

Por lo que concierne a apoyos recibidos, todos los entrevistados coincidieron en que han recibido ayuda financiera del gobierno egipcio y dos mencionaron los servicios de JETRO para contactar clientes en Japón.

En opinión de los entrevistados, las exigencias sanitarias, fitosanitarias y de calidad del producto para poder exportar a Japón son muy rigurosas y superiores a las de otros mercados extranjeros, pero ya están familiarizados con ellas y han logrado satisfacerlas.

Todos los entrevistados emplean semillas de origen egipcio, aunque la tecnología aplicada procede de diversos países, como Bielorrusia, Turquía, Gran Bretaña u otros países de la Unión Europea. 
En todos los casos se mencionó que los clientes extranjeros están interesados en productos orgánicos y que éstos ya se están exportando a Japón, diversos países europeos e incluso a algunos países árabes. Aunque el mercado japonés parece ser muy promisorio, en particular para los productos orgánicos, los exportadores egipcios están conscientes de la fuerte competencia proveniente de algunos países asiáticos, como China e India, así como del reto que representa cumplir con las exigencias de los clientes japoneses, que atañen incluso a todas las operaciones previas al proceso de exportación.

Los entrevistados consideran que para mantenerse en este mercado deben conservar la alta calidad e inocuidad de sus productos, de acuerdo con los estándares japoneses, ofrecer productos orgánicos y cumplir con los plazos de entrega. Además, el suministro se realiza durante todo el año, si bien en la mayoría de los casos en pequeñas cantidades.

Finalmente, los propios entrevistados observaron algunas debilidades que deberán superar los exportadores egipcios si pretenden mantenerse exitosamente en el mercado japonés. Ellas son las siguientes:

- Deficiencias en empaques de alta calidad.

- Débiles vínculos con los grandes compradores en posiciones estratégicas en los mercados japoneses.

- En ocasiones los productos no satisfacen las exigencias de los mercados japoneses, en cuanto a propiedades organolépticas o bien tipo de presentación de la mercancía.

- Competencia proveniente de países localizados más cerca de Japón, como es el caso de China e India, principalmente.

- Desafío de cumplir con estándares en costos, sofisticación del producto, inocuidad alimentaria.

- Falta de personal altamente capacitado para realizar operaciones de exportación y empacado de acuerdo con los estándares de los mercados japoneses.

Los entrevistados opinan que es poco probable que a corto plazo las verduras deshidratadas egipcias puedan imponerse a las de EU y China, pero sí es posible lograr una mayor especialización que les permita aprovechar nichos de mercado para productos con alto valor agregado, aprovechando para ello los contactos que ya existen en el mercado japonés. 


\section{Consideraciones finales}

Nuestra investigación muestra que, a la par con la conformación de grandes centros hegemónicos dentro de la presente globalización, existen esfuerzos importantes por parte de economías ubicadas en uno u otro bloque o espacio dominado por los tres principales hegemones (EU, Unión Europea, ChinaJapón), para diversificar sus vínculos económicos, especialmente comerciales, con el fin de atenuar los lazos de dependencia que los unen a alguno de los centros mencionados.

En particular el mercado japonés representa un escenario de gran interés en el que se despliega una dinámica competencia entre productores-exportadores de los más diversos países. Sin embargo, los desarrollos observados en esta competencia están lejos de poder ser explicados tan solo en términos económicos. Por el contrario, el caso estudiado indica la relevancia de factores históricos, políticos, culturales, demográficos, por mencionar los de mayor peso. Tratándose del producto tomado como eje de nuestro análisis, las cebollas deshidratadas, encontramos los siguientes fenómenos dignos de mención.

El incremento en la demanda de cebolla deshidratada por parte de los fabricantes japoneses de alimentos procesados, obedece a tendencias en los hábitos de consumo de la población japonesa que están dando preferencia a alimentos de preparación fácil y rápida, como sopas, pastas, pizzas, hamburguesas y aderezos, que incluyen entre sus ingredientes principales la cebolla deshidratada. La creciente inclinación por este tipo de alimentos se explica por la conformación de ciertos grupos demográficos y sociales (jóvenes, mujeres trabajadoras, ancianos, personas solteras) cuyas condiciones laborales y familiares los inducen al consumo de cierto tipo de alimentos de manera preferente. Asimismo, debe incluirse como factor relevante la masiva publicidad que en Japón se desarrolla para el consumo de alimentos típicamente occidentales, que encuentra un público receptor en particular dentro de ciertos grupos de edades.

Por el lado de la oferta de cebolla deshidratada se constata que la producción nacional ha sido insuficiente, lo cual está relacionado con las condiciones de la agricultura japonesa en especial a partir de la segunda postguerra, lo cual incluye de manera relevante tanto la escasez como el costo de la mano de obra. Aquí están presentes, por un lado, el envejecimiento de la población ocupada en la agricultura, como la ausencia de un relevo generacional, dado que los hijos de agricultores no aceptan continuar realizando este tipo de labores. 
Para satisfacer esta creciente demanda son imprescindibles las importaciones, dentro de las cuales las egipcias han ido ganando terreno en el mercado japonés. Este dinamismo de la oferta egipcia del producto mencionado no puede explicarse sin las políticas instrumentadas por el gobierno egipcio para la promoción de las exportaciones, ni tampoco sin tomar en cuenta el interés de las empresas egipcias por estar presentes en diversos mercados internacionales.

En suma, el caso estudiado nos muestra una vez más que los fenómenos económicos y su dinámica tienen sus raíces, así como el alcance de sus frutos, más allá de la economía. No tomarlos en cuenta nos pone en peligro de no comprender ni poder explicar nuestro objeto de estudio.

\section{Agradecimientos}

Los autores desean expresar su agradecimiento a las personas entrevistadas, así como a la doctora Kae Sekine de la Universidad de Ryukyu, Okinawa, por los valiosos materiales proporcionados para este trabajo; al licenciado Víctor Nakano de JETRO-México, por sus orientaciones; al señor Kazumi Suhara, Chairman of Japan Dehydrated Vegetable Association y al licenciado Juan José Villarreal Valdez, director de International Sales Sensient Dehydrated Flavors LLC, por compartir con nosotros sus valiosas experiencias.

\section{Referencias bibliográficas}

ALIC (Agriculture \& Livestock Industries Corporation) (2011),たまねぎの受 給動向 (Tendencias de la Oferta y Demanda de Cebollas). Abril. Disponible en: www.vegetable.alic.go.jp/yasaijoho/yasai/1104/yasai1.html. Fecha de consulta: agosto 28, 2012.

Al-Sayyid Marsot, Afat Lufti (2007), A History of Egypt. From the Arab Conquest to the Present, Cambridge: Cambridge University Press.

Altvater, Elmar y Birgit Mahnkopf (2002), Las limitaciones de la globalización. Economía, ecología y política de la globalización, México: Siglo XXI Editores.

Aoki, Sumio Thomas y Paul Miyamoto (2011), "Japan Food Processing Ingredients Food Processing Sector", Global Agricultural Information Network Report, GAIN Report Number: JA 1525, USDA Foreign Agricultural Service. 
Araki, Hajime (2009), Cultivation of Horticulture Crops, Hokkaido University. Disponible: en: http://ocw.hokudai.ac.jp/Course/Faculty/Agriculture/ AgricultureInHokkaido/2009/page/materials/AgricultureInHokkaido2009-Text-03.pdf. Fecha de consulta: febrero 25, 2010.

Bestor, Theodore C. (2006), 'Kaiten-zushi and Konbini: Japanese Food Culture in the Age of Mechanical Reproduction', en Richard Wild (ed.) Fast Food/ Slow Food. The Cultural Economy of the Global Food System, Lanham: AltaMira Press, pp. 115-130.

Hane, Mikiso (2003), Breve historia de Japón, Madrid: Alianza Editorial.

Itoh, Motoshige y Yukiko Ito (2003), 'The Processed Food Industry in Japan', en Japan Food Market Study. A report for the Rural Industries Research and Development Corporation by the Australia-Japan Research Centre (AJRC), Asia Pacific School of Economics and Management (APSEM). RIRDC Publication No. 02/164, RIRDC Project No. ANU-50A.

Mittelman, James H. (2000), The Globalization Syndrome. Transformation and Resistance, Princeton, New Jersey: Princeton University Press.

Pinard, Francois X., Franco Scotti, Morad S. Ahmed (2006), Food Export Strategy Study. Terms of Reference PS 137. Final Report, Egypt, Cairo: IMC.

Tezera, Dejene et al. (2005), Egyptian Processed Food Sector Review. Final Report, Rotterdam: ECORYS-NEI.

Thompson, Jason (2008), A History of Egypt. From Earliest Times to the Present, Cairo: The American University in Cairo Press.

Tsuru, Shigeto (1999), El capitalismo japonés. Algo más que una derrota creativa, Madrid: Akal.

Whitelaw, Gavin Hamilton (2006), 'Rice Ball Rivalries: Japanese Convenience Stores and the Appetite of Late Capitalism', en Richard Wild (ed.) Fast Food/Slow Food. The Cultural Economy of the Global Food System, Lanham: AltaMira Press, pp. 131-144. 